\title{
A polynomial expression for the Hilbert series of the quotient ring of diagonal coinvariants (condensed version)
}

\author{
J. Haglund \\ Department of Mathematics, University of Pennsylvania, Philadelphia, PA, USA
}

\begin{abstract}
A special case of Haiman's identity [Invent. Math. 149 (2002), pp. 371-407] for the character of the quotient ring of diagonal coinvariants under the diagonal action of the symmetric group yields a formula for the bigraded Hilbert series as a sum of rational functions in $q, t$. In this paper we show how a summation identity of Garsia and Zabrocki for Macdonald polynomial Pieri coefficients can be used to transform Haiman's formula for the Hilbert series into an explicit polynomial in $q, t$ with integer coefficients. We also provide an equivalent formula for the Hilbert series as the constant term in a multivariate Laurent series.

Résumé. Un cas spécial de l'identité de Haiman [Invent. Math. 149 (2002), pp. 371-407] pour le caractère de l'anneau quotient des coinvariants diagonaux sous l'action du groupe symétrique fournit une formule pour la série de Hilbert bigraduée comme somme de fonctions rationnelles en $q$, $t$. Dans cet article nous montrons comment une identité de sommation de Garsia et Zabrocki pour les coefficients de Pieri des polynômes de Macdonald peut être utilisée pour transformer la formule de Haiman pour la série de Hilbert en un polynôme explicite en $q, t$ à coefficients entiers. Nous présentons également une formule équivalente pour la série de Hilbert comme terme constant d'une série de Laurent multivariée.
\end{abstract}

Keywords: Hilbert series, diagonal coinvariants

\section{Introduction}

Let $X_{n}=\left\{x_{1}, \ldots, x_{n}\right\}, Y_{n}=\left\{y_{1}, \ldots, y_{n}\right\}$ be two sets of variables and let

$$
\mathrm{DR}_{n}=\mathbb{C}\left[X_{n}, Y_{n}\right] /\left\langle\left\{\sum_{i} x_{i}^{h} y_{i}^{k}, \forall h, k \geq 0, h+k>0\right\}\right\rangle
$$

be the quotient ring of diagonal coinvariants. Let $\nabla$ be the linear operator defined on the modified Macdonald polynomial basis $\left\{\tilde{H}_{\mu}\left(X_{n} ; q, t\right)\right\}$, where $\mu \vdash n$ (i.e. $\mu$ is a partition of $n$ ), by

$$
\nabla \tilde{H}_{\mu}\left(X_{n} ; q, t\right)=T_{\mu} \tilde{H}_{\mu}\left(X_{n} ; q, t\right),
$$

\footnotetext{
${ }^{\dagger}$ Work supported by NSF grant DMS-0901467. jhaglund@math. upenn. edu 
where $T_{\mu}=t^{n(\mu)} q^{n\left(\mu^{\prime}\right)}$ and $n(\mu)=\sum_{i}(i-1) \mu_{i}$. The symmetric group acts "diagonally" on a polynomial $f\left(x_{1}, \ldots, x_{n}, y_{1}, \ldots, y_{n}\right)$ by $\sigma f=f\left(x_{\sigma(1)}, \ldots, x_{\sigma(n)}, y_{\sigma(1)}, \ldots, y_{\sigma(n)}\right)$ and this action extends to $\mathrm{DR}_{n}$. Haiman [Hai02] proved an earlier conjecture of Garsia and Haiman [GH96] that the Frobenius series of this action is given by $\nabla e_{n}\left(X_{n}\right)$, where $e_{n}$ is the $n$th elementary symmetric function in a set of variables. (The Frobenius series is obtained by starting with the character and mapping the irreducible $S_{n}$-character $\chi^{\lambda}$ to the Schur function $s_{\lambda}$.) Since the Frobenius series of $\mathrm{DR}_{n}$ is given by $\nabla e_{n}$, the Hilbert series Hilb $\left(\mathrm{DR}_{n}\right)$ is given by $\left\langle\nabla e_{n}, h_{1}^{n}\right\rangle$ (See [Hag08, p. 24] for an explanation of why. Here $\langle$,$\rangle is the$ Hall scalar product, with respect to which the Schur functions are orthonormal, and $h_{1}(X)=\sum_{i} x_{i}$. This results in a formula for $\mathrm{Hilb}\left(\mathrm{DR}_{n}\right)$ as an explicit sum of rational functions in $q, t$, described in detail in the next section. A corollary of this formula is that $\operatorname{dim}\left(\mathrm{DR}_{n}\right)=(n+1)^{n-1}$. See also [Hai94] and [Ber09] for background on this problem. We mention that many articles in the literature refer to the space of diagonal harmonics $\mathrm{DH}_{n}$, which is known to be isomorphic to $\mathrm{DR}_{n}$, and so $\operatorname{Hilb}\left(\mathrm{DH}_{n}\right)=\operatorname{Hilb}\left(\mathrm{DR}_{n}\right)$.

A Dyck path is a lattice path in the first quadrant of the $x y$-plane from $(0,0)$ to $(n, n)$ consisting of unit north $N$ and east $E$ steps which never goes below the diagonal $x=y$. A parking function is a placement of the integers $1,2, \ldots, n$ (called "cars") just to the right of the $N$ steps of a Dyck path, so there is strict decrease down columns. An open conjecture of Loehr and the author [HL05] expresses Hilb(DR $\left.{ }_{n}\right)$ as a positive sum of monomials, one for each parking function. In a recent preprint, Armstrong [Arm10 introduces a hyperplane arrangement model for $\operatorname{Hilb}\left(\mathrm{DR}_{n}\right)$ involving a pair of hyperplane arrangements with a statistic associated to each one. See also [AR]. He gives a bijection with parking functions which sends his pair of hyperplane arrangement statistics to the pair of statistics on parking functions introduced by Haglund and Loehr.

In this article we use a plethystic summation formula of Garsia and Zabrocki for Macdonald Pieri coefficients to show how $\left\langle\nabla e_{n}, h_{1}^{n}\right\rangle$ can be expressed as an element of $\mathbb{Z}[q, t]$. The most elegant way of expressing our result is to say that $\mathrm{Hilb}\left(\mathrm{DR}_{n}\right)$ is the coefficient of $z_{1} z_{2} \cdots z_{n}$ in a certain multivariate Laurent series (see (41)). We are currently unable to see how our result implies a positive formula such as the conjecture of Haglund and Loehr, but are hopeful that further work will lead to such applications.

\section{Background Material}

For $\mu \vdash n$, and $s$ a square of the Ferrers diagram of $\mu$, let $l(s), a(s), l^{\prime}(s), a^{\prime}(s)$ denote the leg, arm, coleg, coarm, respectively, of $s$, i.e. the number of squares above $s$, to the right of $s$, below $s$, and to the left of $s$, as in Figure 1 Furthermore let

$M=(1-q)(1-t), \quad B_{\mu}=\sum_{s \in \mu} t^{l^{\prime}} q^{a^{\prime}}, \quad \Pi_{\mu}=\prod_{\substack{s \in \mu \\ s \neq(0,0)}}\left(1-t^{l^{\prime}} q^{a^{\prime}}\right), \quad w_{\mu}=\prod_{s \in \mu}\left(q^{a}-t^{l+1}\right)\left(t^{l}-q^{a+1}\right)$.

The known expansion

$$
e_{n}(X)=\sum_{\mu \vdash n} \frac{\tilde{H}_{\mu}(X ; q, t) M \Pi_{\mu} B_{\mu}}{w_{\mu}}
$$




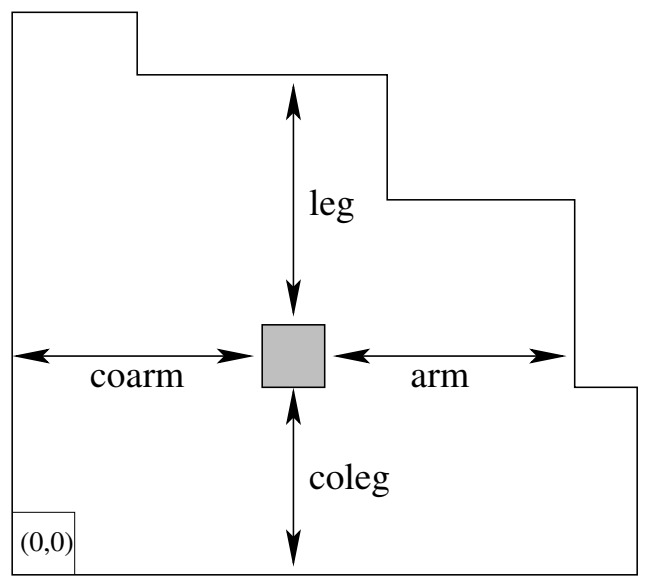

Fig. 1: The leg, coleg, arm, and coarm of a square

then implies

$$
\nabla e_{n}(X)=\sum_{\mu \vdash n} \frac{T_{\mu} \tilde{H}_{\mu}(X ; q, t) M \Pi_{\mu} B_{\mu}}{w_{\mu}} .
$$

Letting $F_{\mu}=\left\langle\tilde{H}_{\mu}, h_{1}^{n}\right\rangle$, by taking the scalar product of both sides of 5 with respect to $h_{1}^{n}$ we get

$$
\operatorname{Hilb}\left(\mathrm{DR}_{n}\right)=\sum_{\mu \vdash n} \frac{T_{\mu} F_{\mu} M \Pi_{\mu} B_{\mu}}{w_{\mu}} .
$$

Let ${ }^{\perp}$ be the operation on symmetric functions which is adjoint to multiplication with respect to the Hall scalar product, i.e. for any symmetric functions $f, g, h$,

$$
\left\langle f^{\perp} g, h\right\rangle=\langle g, f h\rangle .
$$

If $\mu \vdash n$ and $\nu \vdash n-1$, then $\nu \rightarrow \mu$ means $\nu$ is obtained from $\mu$ by removing some corner square of $\mu$, and $\mu \leftarrow \nu$ means $\mu$ is obtained from $\nu$ by adding a single square to the Ferrers shape of $\nu$. Define generalized skew Pieri coefficients $c_{\mu, \nu}^{f^{\perp}}(q, t)$ and Pieri coefficients $d_{\mu, \nu}^{f}(q, t)$ by the formulas

$$
\begin{aligned}
f^{\perp} \tilde{H}_{\mu}(X ; q, t) & =\sum_{\substack{\nu \\
\nu \rightarrow \mu}} c_{\mu, \nu}^{f^{\perp}}(q, t) \tilde{H}_{\nu}(X ; q, t) \\
f \tilde{H}_{\nu}(X ; q, t) & =\sum_{\substack{\mu \\
\mu \leftarrow \nu}} d_{\mu, \nu}^{f}(q, t) \tilde{H}_{\mu}(X ; q, t) .
\end{aligned}
$$

Many of the identities in this paper are expressed using plethystic notation, defined as follows. If $p_{k}(X)=\sum_{i} x_{i}^{k}$ is the $k$ th power sum, then for any expression $E$, the plethystic substitution of $E$ into 
$p_{k}$ is obtained by replacing all indeterminates in $E$ by their $k$ th powers. We denote this by $p_{k}[E]$, so for example

$$
p_{k}[X(1-t)]=p_{k}(X)\left(1-t^{k}\right) .
$$

For any symmetric function $f(X)$, we define $f[E]$ by first expressing $f$ as a polynomial in the $p_{k}$, then replacing each $p_{k}$ by $p_{k}[E]$.

The $c_{\mu, \nu}^{f^{\perp}}$ and the $d_{\mu, \nu}^{f}$ are related via [GH02, (3.5)]

$$
c_{\mu, \nu}^{f^{\perp}} w_{\nu}=d_{\mu, \nu}^{\omega f[X / M]} w_{\mu}
$$

where $\omega$ is the linear operator on symmetric functions satisfying $\omega s_{\lambda}=s_{\lambda^{\prime}}$. Note $d_{\mu, \nu}^{\omega h_{1}[X / M]}=d_{\mu, \nu}^{h_{1}[X]} / M$. We abbreviate $c_{\mu, \nu}^{h_{1} \perp}(q, t)$ by $c_{\mu, \nu}$ and $d_{\mu, \nu}^{h_{1}[X / M]}(q, t)$ by $d_{\mu, \nu}$.

A special case of Macdonald's Pieri formulas [Mac95, Section 6.6] gives an expression for $d_{\mu, \nu}$ as a quotient of factors of the form $\left(t^{a} q^{b}-t^{c} q^{d}\right)$, where $a, b, c, d$ have simple combinatorial descriptions. Garsia found a simplification in this formula, which Garsia and Zabrocki used to obtain the $k=1$ case of the following summation formula [GZ05]. The proof of the result for general $k$ appears in [BGHT99] and [Gar10].

$$
\sum_{\substack{\mu \\ \mu \leftarrow \nu}} d_{\mu, \nu} T^{k}= \begin{cases}1 / M & \text { if } k=0, \\ (-1)^{k-1} e_{k-1}\left[M B_{\nu}-1\right] / M & \text { if } k \geq 1,\end{cases}
$$

where throughout this article $T$ is an abbreviation for $T_{\mu} / T_{\nu}$. Eq. $[12$ is closely related to a corresponding summation formula involving the $c_{\mu, \nu}$ [GT96, Theorem 2.2].

Identity (12) can be recast in the following form.

\section{Lemma 1}

$$
\sum_{\substack{\mu \\
\mu \leftarrow \nu}} d_{\mu, \nu}(1-T) T^{k}=\left\{\begin{array}{ll}
0 & \text { if } k=0, \\
(-1)^{k-1} e_{k}\left[M B_{\nu}\right] / M & \text { if } k \geq 1
\end{array} .\right.
$$

The following simple fact will be useful later.

Lemma 2

$$
(-1)^{k-1} e_{k}[M] / M=\frac{t^{k}-q^{k}}{t-q} \quad k \geq 1 .
$$

\section{A New Recursive Procedure to Generate the Hilbert Series}

By definition we have

$$
e_{1}^{\perp} \tilde{H}_{\mu}(X ; q, t)=\sum_{\substack{\nu \\ \nu \rightarrow \mu}} c_{\mu, \nu} \tilde{H}_{\nu}(X ; q, t) .
$$


Taking the scalar product of both sides with respect to $h_{1}^{n-1}$ we get

$$
\left\langle e_{1}^{\perp} \tilde{H}_{\mu}, h_{1}^{n-1}\right\rangle=\left\langle\tilde{H}_{\mu}, e_{1} h_{1}^{n-1}\right\rangle=F_{\mu}=\sum_{\substack{\nu \\ \nu \rightarrow \mu}} c_{\mu, \nu} F_{\nu} .
$$

Plugging this recurrence for the $F_{\mu}$ into 6 yields

$$
\begin{aligned}
\operatorname{Hilb}\left(\mathrm{DR}_{n}\right) & =\sum_{\mu \vdash n} \frac{T_{\mu} M \Pi_{\mu} B_{\mu}}{w_{\mu}} \sum_{\substack{\nu \\
\nu \rightarrow \mu}} c_{\mu, \nu} F_{\nu} \\
& =\sum_{\nu \vdash n-1} F_{\nu} M \sum_{\substack{\mu \\
\mu \leftarrow \nu}} \frac{B_{\mu} \Pi_{\mu} c_{\mu, \nu} T_{\mu}}{w_{\mu}} .
\end{aligned}
$$

Now from (3) we see

$$
B_{\mu}=B_{\nu}+T, \quad \Pi_{\mu}=\Pi_{\nu}(1-T) .
$$

Using this and the $f=e_{1}$ case of (11) in 18 we get

$$
\operatorname{Hilb}\left(\mathrm{DR}_{n}\right)=\sum_{\nu \vdash n-1} \frac{T_{\nu} F_{\nu} M \Pi_{\nu}}{w_{\nu}} \sum_{\substack{\mu \\ \mu \leftarrow \nu}} d_{\mu, \nu}\left(B_{\nu}+T\right)(1-T) T .
$$

By (13) this implies

$$
\operatorname{Hilb}\left(\mathrm{DR}_{n}\right)=\sum_{\nu \vdash n-1} \frac{T_{\nu} F_{\nu} M \Pi_{\nu}}{w_{\nu}}\left(\frac{e_{1}\left[M B_{\nu}\right]}{M} \frac{e_{1}\left[M B_{\nu}\right]}{M}-\frac{e_{2}\left[M B_{\nu}\right]}{M}\right) .
$$

(Although $e_{1}\left[M B_{\nu}\right] / M$ can be expressed more simply as $e_{1}\left[B_{\nu}\right]$, leaving $[21$ in the above form will prove more useful in the sequel.)

We now iterate the argument; first re-index the sum in 21) as a sum over $\mu \vdash n-1$, and replace $F_{\mu}$ by $\sum_{\nu \rightarrow \mu} c_{\mu, \nu} F_{\nu}$. Then write $B_{\mu}$ as $B_{\nu}+T$ as before, and reverse summation to get

$$
\begin{aligned}
\operatorname{Hilb}\left(\mathrm{DR}_{n}\right) & =\sum_{\nu \vdash n-2} \frac{T_{\nu} F_{\nu} M \Pi_{\nu}}{w_{\nu}} \\
\times & \sum_{\substack{\mu \\
\mu \leftarrow \nu}} d_{\mu, \nu}(1-T) T\left(\frac{e_{1}\left[M\left(B_{\nu}+T\right)\right]}{M} \frac{e_{1}\left[M\left(B_{\nu}+T\right)\right]}{M}-\frac{e_{2}\left[M\left(B_{\nu}+T\right)\right]}{M}\right) .
\end{aligned}
$$

Now for any alphabets $X, Y$ we have

$$
e_{k}[X-Y]=\sum_{j=0}^{k} e_{j}[X] e_{k-j}[-Y]
$$

Hence for $k \geq 1$

$$
(-1)^{k-1} \frac{e_{k}\left[M\left(B_{\nu}+T\right)\right]}{M}=b_{k}+T^{k} a_{k}+\sum_{j=1}^{k-1}-M b_{j} T^{k-j} a_{k-j}
$$


where we have abbreviated $(-1)^{j-1} e_{j}[M] / M$ by $a_{j}$ and $(-1)^{j-1} e_{j}\left[M B_{\nu}\right] / M$ by $b_{j}=b_{j}(\nu)$. Here we have used the fact that $e_{k}[M T] / M=T^{k} e_{k}[M] / M$ (since for any expression $p_{j}[X T]=T^{j} p_{j}[X]$ ). Note also that $a_{1}=1$. The inner sum in (22) thus becomes

$$
\begin{aligned}
\sum_{\substack{\mu \\
\mu \leftarrow \nu}} d_{\mu, \nu}(1-T) T & \left(\left(b_{1}+T a_{1}\right)^{2}+b_{2} a_{1}+T^{2} a_{2}-M b_{1} T a_{1}\right) \\
= & b_{1}^{3}+2 b_{1} a_{1} b_{2}+a_{1}^{2} b_{3}+a_{1} b_{2} b_{1}-M b_{1} a_{1}^{2} b_{2}+a_{1} a_{2} b_{3}
\end{aligned}
$$

by $(13)$.

Let

$$
\begin{aligned}
& A_{1}=b_{1} \\
& A_{2}=b_{1}^{2}+b_{2} a_{1} \\
& A_{3}=b_{1}^{3}+2 b_{1} a_{1} b_{2}+a_{1}^{2} b_{3}+a_{1} b_{2} b_{1}-M b_{1} a_{1}^{2} b_{2}+a_{1} a_{2} b_{3} .
\end{aligned}
$$

The above discussion implies

Theorem 1 For $p \in \mathbb{N}, 1 \leq p \leq n$,

$$
\operatorname{Hilb}\left(D R_{n}\right)=\sum_{\nu \vdash n-p+1} \frac{T_{\nu} F_{\nu} M \Pi_{\nu}}{w_{\nu}} A_{p},
$$

where $A_{p}=A_{p}(\nu)$ is a certain polynomial in the $a_{i}, b_{i}$. Moreover, $A_{p}$ can be calculated recursively from $A_{p-1}$ by the following procedure. First replace each $b_{k}$ in $A_{p-1}$ by $b_{k}+T^{k} a_{k}-\sum_{j=1}^{k-1} M b_{j} T^{k-j} a_{k-j}$. Then multiply the resulting expression out to form a polynomial in $T$, say

$$
\sum_{j} c_{j} T^{j}
$$

Finally, replace $T^{j}$ by $b_{j+1}$, i.e.

$$
A_{p}=\sum_{j} c_{j} b_{j+1}
$$

(We replace $T^{j}$ by $b_{j+1}$ since, after multiplying the expression above out to get $\sum c_{j} T^{j}$, we still have another factor of $T$ coming from the outer sum. Applying $(13)$ replaces $T^{j+1}$ by $b_{j+1}$.)

We now give a non-recursive expression for $A_{p}$. Let $Q_{n}$ denote the set of all $n \times n$ upper-triangular matrices $\mathrm{C}$ of nonnegative integers which satisfy

$$
-\sum_{i=1}^{j-1} c_{i j}+\sum_{i=j}^{n} c_{j i}=1, \quad \text { for each } j, 1 \leq j \leq n .
$$


For example,

$$
\begin{aligned}
Q_{1} & =\{[1]\} \\
Q_{2} & =\left\{\left[\begin{array}{ll}
1 & 0 \\
0 & 1
\end{array}\right],\left[\begin{array}{ll}
0 & 1 \\
0 & 2
\end{array}\right]\right\} \\
Q_{3} & =\left\{\left[\begin{array}{lll}
1 & 0 & 0 \\
0 & 1 & 0 \\
0 & 0 & 1
\end{array}\right],\left[\begin{array}{lll}
1 & 0 & 0 \\
0 & 0 & 1 \\
0 & 0 & 2
\end{array}\right],\left[\begin{array}{lll}
0 & 1 & 0 \\
0 & 2 & 0 \\
0 & 0 & 1
\end{array}\right],\left[\begin{array}{lll}
0 & 1 & 0 \\
0 & 1 & 1 \\
0 & 0 & 2
\end{array}\right],\left[\begin{array}{lll}
0 & 1 & 0 \\
0 & 0 & 2 \\
0 & 0 & 3
\end{array}\right],\left[\begin{array}{lll}
0 & 0 & 1 \\
0 & 1 & 0 \\
0 & 0 & 2
\end{array}\right],\left[\begin{array}{lll}
0 & 0 & 1 \\
0 & 0 & 1 \\
0 & 0 & 3
\end{array}\right]\right\}
\end{aligned}
$$

Geometrically, the condition (33) says that for all $j$, if we add all the entries of $C$ in the $j$ th row together, and then subtract all the entries in the $j$ th column above the diagonal, we get 1 . Note that these conditions imply that each row of $C$ must have at least one positive entry.

For $C \in Q_{n}$, let $\operatorname{Pos}(C)$ denote the multiset of positive entries in $C$, and $\operatorname{pos}(C)$ its cardinality. Matrices of this kind can be generated recursively, in a manner similar to the recursion generating the polynomials $A_{p}$, and using this one can prove the following.

Theorem 2 For $1 \leq p \leq n$ and $A_{p}, b_{j}, a_{j}$ as above,

$$
A_{p}=\sum_{C \in Q_{p}}(-M)^{\operatorname{pos}(C)-n} \prod_{c_{i i} \in \operatorname{Pos}(C)} b_{c_{i i}} \prod_{\substack{c_{i j} \in \operatorname{Pos}(C) \\ i<j}} a_{c_{i j}} .
$$

\section{Corollary 1}

$$
\operatorname{Hilb}\left(D R_{n}\right)=\sum_{C \in Q_{n}}(-M)^{\operatorname{pos}(C)-n} \prod_{\substack{c_{i j} \in \operatorname{Pos}(C) \\ 1 \leq i \leq j \leq n}}\left[c_{i j}\right]_{q, t},
$$

where $[k]_{q, t}=\left(t^{k}-q^{k}\right) /(t-q)$ is the $q$, t-analog of the integer $k$.

Example 1 The weights associated to the elements of $Q_{3}$, listed in the same left-to-right order as in (36) are

$$
1, \quad t+q, \quad t+q, \quad-M(t+q), \quad(t+q)\left(t^{2}+q t+q^{2}\right), \quad t+q, \quad t^{2}+q t+q^{2} .
$$

Thus Hilb $\left(\mathrm{DR}_{3}\right)$ is the sum of these terms, namely

$$
1+2 q+2 t+2 q^{2}+3 q t+2 t^{2}+q^{3}+q^{2} t+q t^{2}+t^{3} .
$$

The sequence $1,2,7,40,357,4820, \ldots$ consisting of the cardinalities of the sets $Q_{1}, Q_{2}, Q_{3}, \ldots$ form entry $A 008608$ in Sloane's on-line encyclopedia of integer sequences. In fact, it was comparing the number of monomials in $A_{n}$ for small $n$ with sequences in Sloane's encyclopedia that led the author to the discovery of the non-recursive expression for the $A_{n}$ in terms of the elements of $Q_{n}$. The sequence was introduced to Sloane's list by Glenn Tesler, who in a private conversation with the author said they arose in unpublished work of Tesler's from the late 1990's on plethystic expressions for Macdonald's $D_{n, r}$ operators. Although Tesler doesn't recall any further details about this work, we will refer to elements of $Q_{n}$ as "Tesler matrices".

The explicit formula 38 for $\operatorname{Hilb}\left(\mathrm{DR}_{n}\right)$ can be formulated as a constant term identity. 
Corollary 2 For $n \geq 1, \operatorname{Hilb}\left(D R_{n}\right)$ is the coefficient of $z_{1} z_{2} \cdots z_{n}$ in

$$
\frac{1}{(-M)^{n}} \prod_{i=1}^{n} \frac{\left(1-z_{i}\right)\left(1-q t z_{i}\right)}{\left(1-q z_{i}\right)\left(1-t z_{i}\right)} \prod_{1 \leq i<j \leq n} \frac{\left(1-z_{i} / z_{j}\right)\left(1-q t z_{i} / z_{j}\right)}{\left(1-q z_{i} / z_{j}\right)\left(1-t z_{i} / z_{j}\right)} .
$$

\section{The $m$-parameter}

The formula $\nabla e_{n}$ for the Frobenius series of $\mathrm{DR}_{n}$ is a special case of a more general result (also due to Haiman [Hai02]) which says that for any positive integer $m, \nabla^{m} e_{n}$ is the Frobenius series of a certain $S_{n}$-module $\mathrm{DR}_{n}^{(m)}$. Hence, from (5) we have

$$
\begin{aligned}
\operatorname{Hilb}\left(\mathrm{DR}_{n}^{(m)}\right) & =\left\langle\nabla^{m} e_{n}, h_{1}^{n}\right\rangle \\
& =\sum_{\substack{\mu \\
\mu \vdash n}} \frac{T_{\mu}^{m} F_{\mu} M \Pi_{\mu} B_{\mu}}{w_{\mu}} .
\end{aligned}
$$

The methods of the previous section can be generalized to show that for any $1 \leq p \leq n$,

$$
\operatorname{Hilb}\left(\mathrm{DR}_{n}^{(m)}\right)=\sum_{\mu \vdash n-p+1} \frac{T_{\nu}^{m} F_{\nu} M \Pi_{\nu}}{w_{\nu}} A_{p}^{(m)},
$$

where $A_{p}^{(m)}=A_{p}^{(m)}(\mu)$ is a polynomial in the $b_{j}, a_{j}$ as before. We have $A_{1}^{(m)}(\mu)=b_{1}$, and for $p>$ 1 , we can construct $A_{p}^{(m)}$ recursively by the following procedure. First, replace each $b_{k}$ in $A_{p-1}^{(m)}$ by $b_{k}+T^{k} a_{k}+\sum_{j=1}^{k-1}-M b_{j} T^{k-j} a_{k-j}$. Then, multiply the resulting expression out to form a polynomial in $T$ say

$$
\sum_{j} c_{j} T^{j}
$$

Finally, replace $T^{j}$ by $b_{j+m}$, i.e.

$$
A_{p}^{(m)}=\sum_{j} c_{j} b_{j+m}
$$

In terms of the Tesler matrices, we want the "hook sums" to be equal to $(1, m, m, \ldots, m)$ instead of $(1,1, \ldots, 1)$. To be precise, define $Q_{n}^{(m)}$ to be the set of upper-triangular matrices $C$ of nonnegative integers satisfying

$$
-\sum_{i=1}^{j-1} c_{i j}+\sum_{i=j}^{n} c_{j i}=\left\{\begin{array}{ll}
1 & \text { if } j=1 \\
m & \text { if } 2 \leq j \leq n
\end{array} .\right.
$$

We get the following extensions of the earlier results. 
Theorem 3 For $1 \leq p \leq n, m \geq 1$, and $A_{p}^{(m)}, b_{j}, a_{j}$ as above,

$$
A_{p}^{(m)}=\sum_{C \in Q_{p}^{(m)}}(-M)^{\operatorname{pos}(C)-n} \prod_{c_{i i} \in \operatorname{Pos}(C)} b_{c_{i i}} \prod_{\substack{c_{i j} \in \operatorname{Pos}(C) \\ i<j}} a_{c_{i j}}
$$

Furthermore, the special case $p=n$ of $(44)$ reduces to

$$
\operatorname{Hilb}\left(D R_{n}^{(m)}\right)=\sum_{C \in Q_{n}^{(m)}}(-M)^{p o s(C)-n} \prod_{\substack{c_{i j} \in \operatorname{Pos}(C) \\ 1 \leq i \leq j \leq n}}\left[c_{i j}\right]_{q, t} .
$$

Corollary 3 For $n \geq 1$, Hilb $\left(D R_{n}^{(m)}\right)$ is the coefficient of $z_{1} z_{2}^{m} z_{3}^{m} \cdots z_{n}^{m}$ in

$$
\frac{1}{(-M)^{n}} \prod_{i=1}^{n} \frac{\left(1-z_{i}\right)\left(1-q t z_{i}\right)}{\left(1-q z_{i}\right)\left(1-t z_{i}\right)} \prod_{1 \leq i<j \leq n} \frac{\left(1-z_{i} / z_{j}\right)\left(1-q t z_{i} / z_{j}\right)}{\left(1-q z_{i} / z_{j}\right)\left(1-t z_{i} / z_{j}\right)}
$$

\section{Conjectures and Open Questions}

\subsection{Tesler matrices with more general hook sums}

In general the coefficient of $z_{1}^{\alpha_{1}} z_{2}^{\alpha_{2}} \cdots z_{n}^{\alpha_{n}}$ in 50 is not a positive polynomial in $q$, $t$, but Maple calculations suggest it is positive if the $\alpha_{i}$ are positive and nondecreasing.

Conjecture 1 For $n \geq 1$ and $\alpha$ the reverse of a partition ( $\operatorname{so} 1 \leq \alpha_{1} \leq \alpha_{2} \leq \cdots \leq \alpha_{n}$ )

$$
\left.\frac{1}{(-M)^{n}} \prod_{i=1}^{n} \frac{\left(1-z_{i}\right)\left(1-q t z_{i}\right)}{\left(1-q z_{i}\right)\left(1-t z_{i}\right)} \prod_{1 \leq i<j \leq n} \frac{\left(1-z_{i} / z_{j}\right)\left(1-q t z_{i} / z_{j}\right)}{\left(1-q z_{i} / z_{j}\right)\left(1-t z_{i} / z_{j}\right)}\right|_{z_{1}^{\alpha_{1}} z_{2}^{\alpha_{2}} \ldots z_{n}^{\alpha_{n}}} \in \mathbb{N}[q, t] .
$$

Equivalently, the weighted sum over Tesler matrices with hook sums $\alpha_{1}, \ldots, \alpha_{n}$ is in $\mathbb{N}[q, t]$.

Remark 1 The argument proving Theorem 3 shows that if $\alpha_{1}=1$, the coefficient of $z_{1}^{\alpha_{1}} z_{2}^{\alpha_{2}} \cdots z_{n}^{\alpha_{n}}$ in (51) can be obtained by starting with $\nabla^{\alpha_{2}} e_{n}$, applying $e_{1}^{\perp}$, then applying $\nabla^{\alpha_{3}-\alpha_{2}}$, applying $e_{1}^{\perp}$ again, then applying $\nabla^{\alpha_{4}-\alpha_{3}}$, etc. The author doesn't know if these polynomials have a representation-theoretic interpretation for $\alpha \neq(1, m, m, \ldots, m)$.

\subsection{A refinement of the $q, t$-positivity}

Note that $[k]_{q, t}$ can be expressed as $s_{k-1}(\{q, t\})$, i.e. the $(k-1)$ st complete homogeneous symmetric function evaluated in the set of variables $\{q, t\}$. Also, $-M=t+q-1-q t$ equals $s_{1}-1-s_{1,1}$, also in the set of variables $\{q, t\}$. In $\left[38\right.$ we can substitute in these Schur function formulations for $[k]_{q, t}$ and $-M$, multiply everything out using the Pieri rule for Schur function multiplication, and thereby obtain a formula for $\operatorname{Hilb}\left(\mathrm{DR}_{n}\right)$ in terms of Schur functions in the set of variables $\{q, t\}$. If we then cancel terms of the form $s_{\lambda}$ where $\lambda$ has more than two parts (which becomes zero since our set of variables has only two elements) it appears that the resulting expression is Schur-positive. For example, for $n=3$ the terms from (39) become

$$
1, s_{1}, \quad s_{1}, \quad\left(s_{1}-1-s_{1,1}\right) s_{1}, \quad s_{1} s_{2}, \quad s_{1}, \quad s_{2},
$$


and the sum of these equals $1+2 s_{1}+2 s_{2}+s_{1,1}+s_{2}-s_{1,1,1}+s_{3}$. Since $s_{1,1,1}(\{q, t\})=0$, we can remove this leaving

$$
\operatorname{Hilb}\left(\mathrm{DR}_{3}\right)=1+2 s_{1}+2 s_{2}+s_{1,1}+s_{2}+s_{3} .
$$

F. Bergeron [Ber09, p.196] has previously conjectured a stronger statement, namely that

$$
\operatorname{Hilb}\left(\mathrm{DR}_{n}\right)=\sum_{\sigma \in S_{n}} h_{\lambda(\sigma)}(\{q, t\})
$$

i.e. that for each permutation on $n$ elements, there is some way of defining a partition $\lambda(\sigma)$ such that the sum of the $h_{\lambda(\sigma)}$ gives Hilb $\left(\mathrm{DR}_{n}\right)$. Here $h_{\lambda}=\prod_{i} s_{\lambda_{i}}$ as before. When $n=3$, the expansion is

$$
\operatorname{Hilb}\left(\mathrm{DR}_{3}\right)=1+2 h_{1}+h_{2}+h_{1,1}+h_{3},
$$

in agreement with 53 . Bergeron further conjectures that these sums have the remarkable property that if we evaluate them in the set of variables $\left\{q_{1}, q_{2}, \ldots, q_{k}\right\}$ we get the Hilbert series of diagonal coinvariants in $k$ sets of variables, for any $k \geq 1$. We hope that further study of how the cancellation in identity (38) results in positivity will lead to progress on the $k=2$ case of Bergeron's conjecture.

\section{References}

[AR] D. Armstrong and B. Rhoades. The Shi arrangement and the Ish arrangement. Trans. Amer. Math. Soc. to appear, preprint at http://www.math.miami.edu/ armstrong/research.html.

[Arm10] D. Armstrong. Hyperplane arrangements and diagonal harmonics. Preprint at http://www.math.miami.edu/ armstrong/research.html, 2010.

[Ber09] François Bergeron. Algebraic combinatorics and coinvariant spaces. CMS Treatises in Mathematics. Canadian Mathematical Society, Ottawa, ON, 2009.

[BGHT99] F. Bergeron, A. M. Garsia, M. Haiman, and G. Tesler. Identities and positivity conjectures for some remarkable operators in the theory of symmetric functions. Methods Appl. Anal., 6:363-420, 1999.

[Gar10] A. Garsia. The $d_{\mu, \nu}$ summation formula. Unpublished note, 2010.

[GH96] A. M. Garsia and M. Haiman. A remarkable $q, t$-Catalan sequence and $q$-Lagrange inversion. J. Algebraic Combin., 5(3):191-244, 1996.

[GH02] A. M. Garsia and J. Haglund. A proof of the $q, t$-Catalan positivity conjecture. Discrete Math., 256:677-717, 2002.

[GT96] A. M. Garsia and G. Tesler. Plethystic formulas for Macdonald $q, t$-Kostka coefficients. Adv. Math., 123:144-222, 1996.

[GZ05] A. Garsia and M. Zabrocki. Some calculations for the outer hookwalk. Unpublished note, 2005. 
[Hag08] James Haglund. The q,t-Catalan numbers and the space of diagonal harmonics, volume 41 of University Lecture Series. American Mathematical Society, Providence, RI, 2008. With an appendix on the combinatorics of Macdonald polynomials.

[Hai94] M. Haiman. Conjectures on the quotient ring by diagonal invariants. J. Algebraic Combin., 3:17-76, 1994.

[Hai02] M. Haiman. Vanishing theorems and character formulas for the Hilbert scheme of points in the plane. Invent. Math., 149:371-407, 2002.

$\left[\mathrm{HHL}^{+}\right.$05] J. Haglund, M. Haiman, N. Loehr, J. B. Remmel, and A. Ulyanov. A combinatorial formula for the character of the diagonal coinvariants. Duke J. Math., 126:195-232, 2005.

[HL05] J. Haglund and N. Loehr. A conjectured combinatorial formula for the Hilbert series for Diagonal Harmonics. Discrete Math. (Proceedings of the FPSAC 2002 Conference held in Melbourne, Australia), 298:189-204, 2005.

[Mac95] I. G. Macdonald. Symmetric Functions and Hall Polynomials. Oxford Mathematical Monographs, second ed., Oxford Science Publications. The Clarendon Press Oxford University Press, New York, 1995. 
